\title{
Impacto do Uso Prolongado da Terapêutica Subcutânea Contínua com Insulina no Controlo da Diabetes Mellitus Tipo 1
}

\section{The Impact of Prolonged Use of Continuous Subcutaneous Insulin Infusion in the Control of Type-1 Diabetes}

Sérgio AZEVEDO $\otimes^{1}$, Joana SARAIVA ${ }^{1,2}$, Francisco CARAMELO',3, Lúcia FADIGA², Luísa BARROS ${ }^{2}$, Carla BAPTISTA², Miguel MELO ${ }^{1,2}$, Leonor GOMES 1,2 , Francisco CARRILHO 2

Acta Med Port 2019 Jan;32(1):17-24 - https://doi.org/10.20344/amp.10778

\section{RESUMO}

Introdução: O uso da terapêutica com perfusão subcutânea contínua de insulina na diabetes mellitus tipo 1 é cada vez mais frequente devido aos seus efeitos benéficos no controlo glicémico e na flexibilidade do estilo de vida. Constituiu objetivo deste estudo avaliar o impacto da terapêutica com perfusão subcutânea contínua de insulina no controlo glicémico, índice de massa corporal, dose diária total de insulina e complicações desta modalidade terapêutica durante vinte anos de experiência no Centro Hospitalar e Universitário de Coimbra.

Material e Métodos: Estudo retrospetivo que inclui doentes com diabetes mellitus tipo 1 seguidos no Centro Hospitalar e Universitário de Coimbra, que iniciaram terapêutica com perfusão subcutânea contínua de insulina até 2005 e com pelo menos 10 anos de tratamento com terapêutica com perfusão subcutânea contínua de insulina. Avaliou-se a hemoglobina glicada A1c, o índice de massa corporal e a dose diária total de insulina imediatamente antes e seis meses, um ano, cinco, 10, 15 e 20 anos após terapêutica com perfusão subcutânea contínua de insulina a partir dos registos médicos. Avaliou-se ainda a frequência de complicações agudas associadas a este tipo de terapêutica.

Resultados: Obtiveram-se dados de 20 doentes (sete homens; 13 mulheres) com duração média de doença até início da terapêutica com perfusão subcutânea contínua de insulina de 16,1 $\pm 7,9$ anos, idade média de início de terapêutica com perfusão subcutânea contínua de insulina de 31,1 $\pm 8,4$ anos e seguimento durante 13,2 $\pm 2,3$ anos. As indicações para colocação de bomba foram: inadequado controlo metabólico em 15 doentes, história de hipoglicemias assintomáticas ou severas em quatro doentes, e gravidez/ planeamento de gravidez em um doente. A mediana de hemoglobina glicada A1c prévia foi 9,3\% (6,5 - 16,0) tendo diminuído aos seis meses para o valor mínimo de 7,2\% (5,3-9,8); $p<0,0125$. A redução da hemoglobina glicada A1c manteve-se estatisticamente significativa nos primeiros 10 anos de seguimento. Verificou-se uma diferença estatisticamente significativa na variação do índice de massa corporal após 10 anos de seguimento comparativamente com o valor prévio à terapêutica com perfusão subcutânea contínua de insulina; $24,7 \mathrm{~kg} / \mathrm{m}^{2}(18,9-31,8)$ vs $25,5 \mathrm{~kg} / \mathrm{m}^{2}(18,9-38,9), p<0,0125$. As necessidades diárias de insulina foram reduzidas de $56,5 \cup(32,0$ - 94,0) para 43,8 U $(33,0$ - 64,0) $(p<0,0125)$ nos primeiros seis meses e não se encontraram diferenças estatísticas no restante seguimento relativamente às necessidades prévias à terapêutica com perfusão subcutânea contínua de insulina. Verificaramse duas hipoglicemias severas (incidência 0,0095/doente/ano), cinco cetoacidoses diabéticas (0,0238/doente/ano) e nenhuma infeção no local de inserção do cateter.

Discussão: Este estudo demonstrou a eficácia da terapêutica com perfusão subcutânea contínua de insulina, que está associada a uma diminuição significativa da hemoglobina glicada A1c sustentada durante 10 anos e a uma redução da dose diária total de insulina, significativa nos primeiros seis meses. A taxa de complicações agudas foi baixa.

Conclusão: A evidência sugere que a terapêutica com perfusão subcutânea contínua de insulina é efetivamente vantajosa no controlo metabólico em doentes com diabetes mellitus tipo 1 selecionados.

Palavras-chave: Diabetes Mellitus Tipo 1; Hemoglobina Glicada A1c; Infusões Subcutâneas; Insulina; Sistemas de Perfusão de Insulina

\section{ABSTRACT}

Introduction: The use of continuous subcutaneous insulin infusion therapy in type 1 diabetes mellitus has increased due to its benefits on glycemic control and on the lifestyle flexibility. The aim of this study was to assess the impact of continuous subcutaneous insulin infusion therapy on glycemic control, body mass index, total daily dose of insulin and complications associated with this therapy, during 20 years of experience in Centro Hospitalar e Universitário de Coimbra.

Material and Methods: This retrospective study included patients with type 1 diabetes mellitus who started continuous subcutaneous insulin infusion therapy up until 2005, followed at Centro Hospitalar e Universitário de Coimbra. Glycated hemoglobin A1c, body mass index, total daily dose of insulin and acute complications associated with continuous subcutaneous insulin infusion therapy were evaluated immediately prior to initiation of continuous subcutaneous insulin infusion therapy with follow-up at six months, one year, five, 10, 15 and 20 years. The frequency of acute complications associated with this type of therapy was also evaluated.

Results: This study included 20 patients (seven males, 13 females) with mean disease duration up to the start of continuous subcutaneous insulin infusion therapy of $16.1 \pm 7.9$ years, mean age of onset of continuous subcutaneous insulin infusion therapy of $31.1 \pm 8.4$ years and follow-up during $13.2 \pm 2.3$ years. The reasons for initiating pump therapy were: inadequate metabolic control in 15 patients, history of asymptomatic or severe hypoglycemia in four patients, and pregnancy/pregnancy planning in one patient. The

1. Faculdade de Medicina. Universidade de Coimbra. Coimbra. Portugal.

2. Serviço de Endocrinologia, Diabetes e Metabolismo. Centro Hospitalar e Universitário de Coimbra. Coimbra. Portugal.

3. Laboratório de Bioestatística e Informática Médica. Instituto Biomédico de Investigação da Luz e da Imagem. Coimbra. Portugal.

$\square$ Autor correspondente: Sérgio Azevedo. sergiommazevedo@hotmail.com

Recebido: 11 de maio de 2018 - Aceite: 31 de outubro de 2018 | Copyright $\odot$ Ordem dos Médicos 2019 
previous median of glycated hemoglobin A1c was $9.3 \%(6.5-16.0)$ and, at six months, decreased to the minimum value of $7.2 \%(5.3-$ $9.8) ; p<0.0125$. The reduction of glycated hemoglobin A1c remained statistically significant in the first 10 years of follow-up. There was a statistically significant difference in the body mass index variation at 10 years with continuous subcutaneous insulin infusion therapy compared to previous body mass index; $24.7 \mathrm{~kg} / \mathrm{m}^{2}(18.9-31.8) \mathrm{vs} 25,5 \mathrm{~kg} / \mathrm{m}^{2}(18.9-38.9), p<0.0125$. Daily insulin requirements were reduced from $56.5 \mathrm{U}(32.0-94.0)$ to $43.8 \mathrm{U}(33.0-64.0)(p<0.0125)$ at six months and no statistical differences were found in the remaining follow-up. There were two severe episodes of hypoglycemia (incidence 0.0095/patient/year), five episodes of diabetic ketoacidosis (0.0238/patient/year) and no infections at the site of catheter insertion.

Discussion: This study shows that continuous subcutaneous insulin infusion therapy improved glycemic control, especially during the first 10 years of follow-up and allowed a significant decrease in total daily dose of insulin in the first six months. The rate of acute complications was low.

Conclusion: Treatment with continuous subcutaneous insulin infusion therapy seems effective in achieving metabolic control in selected patients with type 1 diabetes mellitus.

Keywords: Diabetes Mellitus, Type 1; Glycated Hemoglobin A; Infusions, Subcutaneous; Insulin Infusion Systems; Insulin

\section{INTRODUÇÃO}

A diabetes mellitus (DM) é um conjunto de distúrbios no metabolismo caracterizado por hiperglicemia resultante de um défice de secreção de insulina, resistência à ação da insulina ou ambos. ${ }^{1}$

Como consequência da hiperglicemia crónica poderão surgir complicações microvasculares e macrovasculares. Nos doentes com diabetes mellitus tipo 1 (DM1), diversos estudos mostram que um tratamento intensivo, de forma a manter níveis glicémicos próximos da normalidade, reduz o risco de complicações a longo prazo. ${ }^{2,3}$ De forma a obter um controlo glicémico otimizado, é necessário recorrer a uma terapêutica de substituição que mimetize o perfil de produção de insulina de um indivíduo sem diabetes. A insulinoterapia com múltiplas administrações diárias (MAD) e a terapêutica de perfusão subcutânea contínua de insulina (PSCl) constituem duas opções eficazes. ${ }^{4}$

A terapêutica através de $\mathrm{PSCl}$, também conhecida como bomba infusora de insulina (BII), foi introduzida há quase meio século tendo evoluído desde então. As primeiras bombas infusoras de insulina eram máquinas volumosas utilizadas apenas para fins de investigação. Desde então as capacidades tecnológicas destes sistemas evoluíram drasticamente e atualmente o tamanho de uma bomba infusora de insulina não ultrapassa o de um telemóvel. ${ }^{5} \mathrm{O}$ sistema de infusão é constituído por uma bomba eletromecânica portátil que contém um reservatório de insulina preenchido com análogo de insulina de ação rápida. $A$ insulina é difundida através de um cateter chegando ao tecido celular subcutâneo por uma cânula aí implantada. Este sistema permite a perfusão subcutânea de insulina com um débito basal contínuo pré-definido nas 24 horas e bólus ativados pelo doente às refeições ou sempre que as glicémias o justificarem. ${ }^{6}$
A Direção Geral de Saúde ${ }^{7}$ definiu que uma pessoa com diabetes, para se tornar elegível para tratamento através de PSCl, deverá possuir motivação e prática de auto monitorização da glicémia capilar, competência na sua utilização de forma satisfatória e possuir requisitos específicos, os quais são enumerados na Tabela 1.

No que diz respeito à eficácia da $\mathrm{PSCl}$, a maioria dos estudos existentes são comparações diretas das MAD com a PSCI. Na revisão de Pozzilli et al, ${ }^{8}$ que reúne o resultado de múltiplas meta-análises e revisões sistemáticas, foi relatado uma superioridade da $\mathrm{PSCl}$ na redução da hemoglobina glicada $\mathrm{A} 1 \mathrm{c}(\mathrm{HbA} 1 \mathrm{c})$ tanto em adultos (com redução da $\mathrm{HbA} 1 \mathrm{c}$ de $0,29 \%$ ) como em crianças (redução de $0,22 \%$ da HbA1c) com DM1. Além da melhoria do controlo glicémico após introdução da $\mathrm{PSCl}$, que é mais marcado em indivíduos com pior controlo glicémico prévio, verificou-se uma necessidade menor das doses diárias de insulina comparativamente com as MAD. Atualmente é largamente recomendado o uso da $\mathrm{PSCl}$ em doentes selecionados com DM1.8

São poucos os estudos que avaliam a eficácia da terapêutica com $\mathrm{PSCl}$ no controlo glicémico a longo-prazo. Alguns estudos demonstram uma redução significativa da HbA1c até 10 anos após início da terapêutica com PSCI. ${ }^{9-12}$ Estudos portugueses, com uma duração máxima de cinco anos, revelam uma melhoria do controlo glicémico, especialmente nos primeiros seis meses de terapêutica. Após os seis meses, o comportamento da $\mathrm{HbA} 1 \mathrm{c}$ é variável, com estudos a reportar uma redução significativa durante todo o seguimento ${ }^{4,13,14}$ e outros em que esta redução significativa a longo-prazo não se verifica. ${ }^{15,16}$

Relativamente às complicações agudas associadas à terapêutica com $\mathrm{PSCl}$, o estudo DCCT mostrou um risco

Tabela 1 - Elegibilidade dos doentes para tratamento com PSCI

\section{Pelo menos um dos seguintes requisitos:}

1. Controlo metabólico não aceitável a fazer insulinoterapia intensiva com múltiplas administrações de insulina (pelo menos 4 administrações/dia) incluindo insulina glargina ou outra com idêntico perfil farmacocinético, definido como:

a. $\mathrm{HbA} 1 \mathrm{c}>7 \%$;

b. Fenómeno do alvorecer com níveis de glicémia > 140 - $160 \mathrm{mg} / \mathrm{dL}$;

c. Acentuada variabilidade diária nos níveis de glicemia.

2. História de hipoglicemia sem pródromos ou hipoglicemias severas frequentes

3. Necessidade de flexibilidade no estilo de vida

4. Gravidez (ou planeamento da gravidez)

5. Necessidade de pequenas doses de insulina

Adaptado de: Direção Geral de Saúde. Circular normativa nº17/DSCS/DGDID. 2008 
aumentado de hipoglicemia como consequência do tratamento da DM1 com insulinoterapia intensiva. ${ }^{2}$ Alguns estudos mais recentes mostram uma menor frequência de hipoglicémia severa em doentes que passaram de terapêutica com MAD para PSCI. ${ }^{13,17,18}$ Em termos de cetoacidose diabética, os primeiros estudos reportaram um aumento da sua frequência com a utilização de $\mathrm{PSCl} .{ }^{5}$ Isto seria devido ao funcionamento errático da bomba perfusora de insulina e inadequada educação tanto do doente como dos profissionais de saúde. ${ }^{5}$ Estudos mais recentes, que comparam as MAD com a PSCl, mostram que a frequência desta complicação na $\mathrm{PSCl}$ é semelhante ou inferior comparativamente às MAD. ${ }^{6}$

Com este estudo pretende-se avaliar o impacto da utilização da $\mathrm{PSCl}$ a longo prazo no controlo glicémico, índice de massa corporal (IMC), dose diária total de insulina (DDTI) e frequência de complicações relacionados com este tipo de tratamento, nomeadamente hipoglicemia severa, cetoacidose diabética e infeções recorrentes no local de inserção do cateter.

\section{MATERIAL E MÉTODOS}

Trata-se de um estudo retrospetivo e observacional que pretendeu avaliar a evolução do controlo glicémico, IMC e DDTI após o início da terapêutica com PSCI.

Foram incluídos os doentes com DM1 que iniciaram terapêutica com $\mathrm{PSCl}$ até dezembro de 2005 no Centro Hospitalar e Universitário de Coimbra (CHUC), tendo estado anteriormente sob terapêutica com MAD. Foram excluídos os casos que não cumpriam pelo menos 10 anos de seguimento em consulta externa no Serviço de Endocrinologia, Diabetes e Metabolismo do CHUC bem como aqueles que tinham iniciado terapêutica com PSCl antes dos 18 anos de idade.

Avaliou-se a HbA1c, o IMC e a DDTI imediatamente antes do início da terapêutica com PSCl e seis meses, um ano, cinco, 10, 15 e 20 anos após $\mathrm{PSCl}$ a partir dos registos médicos das consultas de acompanhamento no CHUC.

Relativamente à frequência de complicações agudas, foi avaliada a ocorrência de cetoacidose diabética, hipoglicemia severa e infeção do local de inserção do cateter após início da terapêutica. Para tal, foi considerado como um episódio de cetoacidose diabética todas as situações em que o doente se apresentava com glicemia superior a 250 $\mathrm{mg} / \mathrm{dL}$ e associadamente bicarbonato sérico $<15 \mathrm{mEq} / \mathrm{L}$ ou $\mathrm{pH}<7,3$ e cetonemia ou cetonúria necessitando de cuidados de saúde urgentes. Hipoglicémia severa foi conside- rada sempre que existiu um episódio com sintomatologia característica de hipoglicemia requerendo necessidade de assistência por outra pessoa para a sua reversão, de preferência acompanhado por uma glicemia confirmada $<50$ $\mathrm{mg} / \mathrm{dL}$.

A análise estatística foi realizada recorrendo ao software Statistical Package for the Social Sciences (SPSS) versão 22. A análise estatística consistiu na comparação das variáveis $\mathrm{HbA} 1 \mathrm{c}$, IMC e DDTI ao fim de seis meses, um ano, cinco anos e 10 anos com o valor pré- $\mathrm{PSCl}(0)$. O período correspondente a 15 anos de seguimento foi excluído do tratamento inferencial de dados devido ao valor reduzido do tamanho da amostra $(n=4)$. Para comparação das variáveis emparelhadas foi utilizado o teste não paramétrico de Wilcoxon. Foi aplicada a correção de Bonferroni de forma a diminuir o erro associado a múltiplas comparações. Este método de correção define um nível de significância a considerar em cada comparação de acordo com o número de comparações efetuadas no total. Considerando um intervalo de confiança de 95\% e aplicando a correção de Bonferroni, sendo que para cada variável existirão quatro comparações efetuadas no total, um teste é estatisticamente significativo se $p<0,0125$. Os resultados são apresentados como mediana (mínimo - máximo) para variáveis contínuas e como frequência absoluta (relativa, \%) para variáveis categóricas.

\section{RESULTADOS}

A amostra incluiu 20 doentes com idades compreendidas entre os 31 e os 61 anos, sete (35\%) do sexo masculino e $13(65 \%)$ do sexo feminino, cujas características clínicas estão presentes na Tabela 2.

A principal indicação para início da terapêutica com PSCl foi controlo metabólico não aceitável em 15 (75\%) doentes. Em quatro (20\%) dos doentes optou-se por este método terapêutico devido a história de hipoglicemia sem pródromos ou hipoglicemias severas frequentes e uma $(5 \%)$ doente devido a gravidez (ou planeamento de gravidez).

A Fig. 1 representa a evolução do valor de $\mathrm{HbA} 1 \mathrm{c}$ da amostra, através de diagramas de extremos e quartis. A $\mathrm{HbA} 1$ c prévia à colocação da $\mathrm{PSCl}$ era de $9,3 \%(6,5-16,0)$ tendo diminuído para $7,2 \%(5,3-9,8)$ após seis meses de terapêutica; $p<0,0125$. Ao fim de um ano a $\mathrm{HbA} 1 \mathrm{c}$ era de $7,6 \%(5,4-8,9)(p<0,0125)$ e aos cinco anos a HbA1c foi de $7,6 \%(6,1-9,0)(p<0,0125)$. Esta redução manteve-se estatisticamente significativa aos 10 anos de seguimento;

Tabela 2 - Caracterização da amostra

\begin{tabular}{lccc}
\hline & M \pm DP & Mín. & Máx. \\
\hline Idade (anos) & $44,3 \pm 8,7$ & 31,0 & 61,0 \\
Idade de diagnóstico (anos) & $15,0 \pm 9,9$ & 1,0 & 37,0 \\
Duração da diabetes até colocação de PSCI (anos) & $16,1 \pm 7,9$ & 4,0 & 32,0 \\
Idade de colocação de PSCI (anos) & $31,1 \pm 8,4$ & 18,0 & 48,0 \\
Duração da terapêutica com PSCI (anos) & $13,2 \pm 2,3$ & 12,0 & 21,0 \\
\hline
\end{tabular}

M \pm DP: média \pm desvio-padrão; Mín.: mínimo; Máx.: máximo 


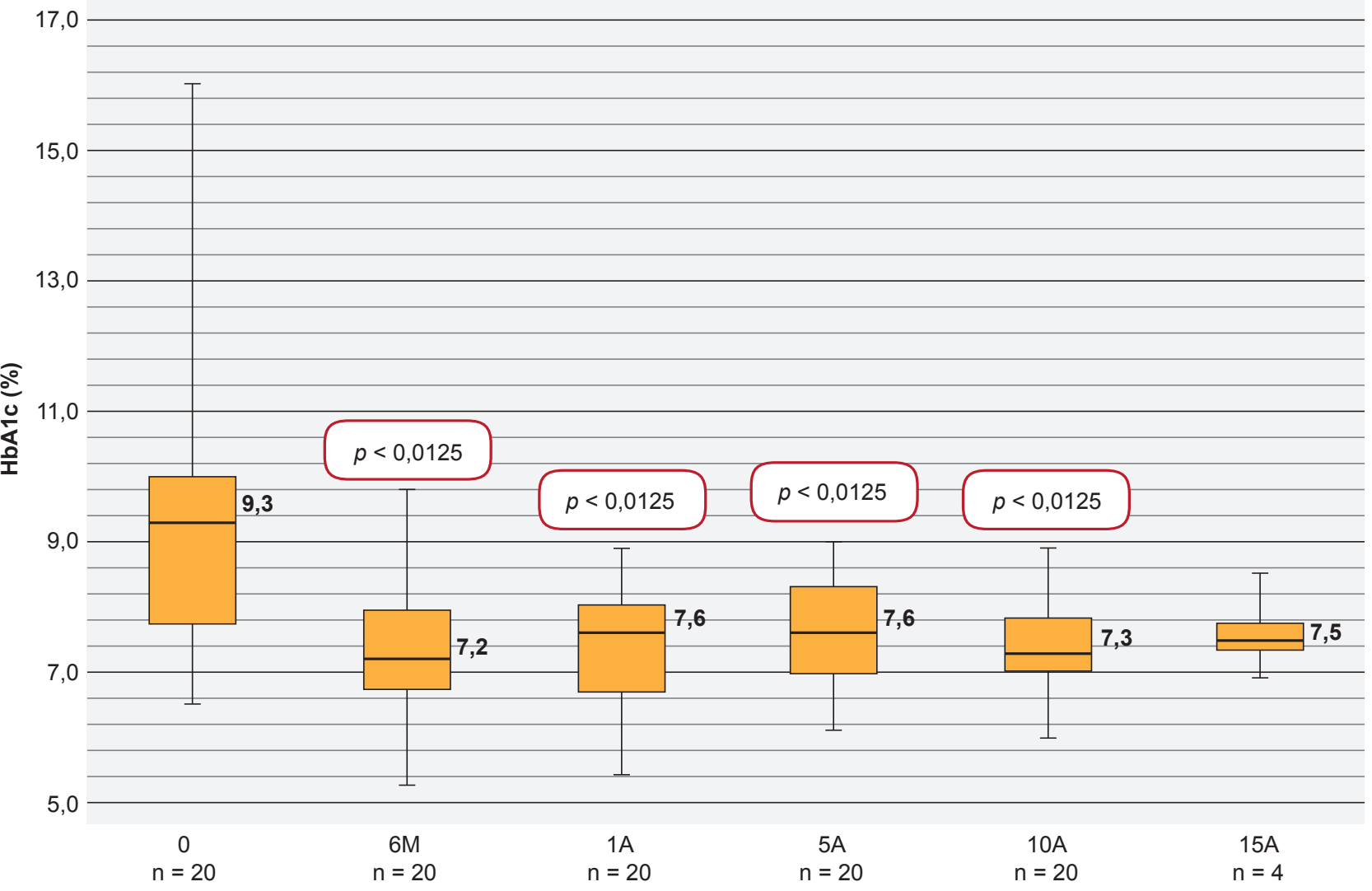

Figura 1 - Boxplots - Evolução da HbA1c. Estatisticamente significativo se $p<0,0125$.

HbA1c: hemoglobina glicada A1c; M: meses; A: anos

$7,3 \%(6,0-8,9), p<0,0125$. Aos 15 anos o tamanho da amostra foi reduzido ( $n=4)$, apresentando um valor de $\mathrm{HbA} 1 \mathrm{c}$ de $7,5 \%(6,9-8,5)$.

O primeiro sistema de $\mathrm{PSCl}$ a ser aplicado na nossa instituição foi há mais de 20 anos. A HbA1c prévia do doente em questão era de $16,0 \%$, verificando-se uma redução marcada da $\mathrm{HbA} 1 \mathrm{c}$ após implementação da $\mathrm{PSCl}$, com uma $\mathrm{HbA} 1 \mathrm{c}$ aos seis meses de $8,9 \%$ e um valor mínimo de $\mathrm{HbA} 1 \mathrm{c}$ de $8,0 \%$ ao fim do primeiro ano. Nos anos consecutivos verifica-se uma ligeira variação da $\mathrm{HbA} 1 \mathrm{c}$ atingindo, ao fim de 20 anos de terapêutica com $\mathrm{PSCl}$, uma $\mathrm{HbA} 1 \mathrm{c}$ de 9,0\% (Fig. 2).

A Fig. 3 representa a variação do IMC ao longo do seguimento. O IMC antes do início de PSCI era de $24,7 \mathrm{~kg} / \mathrm{m}^{2}$ $(18,9$ - 31,8) observando-se um aumento nos primeiros seis meses para $27,8 \mathrm{~kg} / \mathrm{m}^{2}(23,3-33,3) ; p=0,3743$. Ao fim do primeiro ano após início de PSCI, observou-se um IMC de $24,2 \mathrm{~kg} / \mathrm{m}^{2}(19,4-36,7) ; p=0,1823$. Aos cinco anos a variação do IMC relativamente ao valor prévio continua sem atingir significado estatístico; $24,9 \mathrm{~kg} / \mathrm{m}^{2}(18,6-38,1), p=$ 0,0159 . Regista-se apenas uma diferença significativa do IMC aos 10 anos de seguimento comparativamente ao IMC antes da implementação de PSCl; $25,5 \mathrm{~kg} / \mathrm{m}^{2}(18,9$ - 38,9), $p<0,0125$. O tamanho da amostra foi muito reduzido aos 15 anos de seguimento ( $n=4)$, obtendo-se um IMC de 25,8 $\mathrm{kg} / \mathrm{m}^{2}(23,1$ - 34,2). O IMC do indivíduo com 20 anos de seguimento previamente à $\mathrm{PSCl}$ era de $24,7 \mathrm{~kg} / \mathrm{m}^{2}$ tendo atingido um IMC máximo após seis meses de terapêutica com PSCl de $28,4 \mathrm{~kg} / \mathrm{m}^{2}$ e um IMC mínimo de $24,2 \mathrm{~kg} / \mathrm{m}^{2}$ após 20 anos sob terapêutica com PSCl.

A Fig. 4 ilustra a evolução da DDTI após instituição da terapêutica com PSCI. A DDTI foi reduzida significativamente nos primeiros seis meses de terapêutica com PSCI relativamente à DDTI prévia; $56,5 \mathrm{U}(32,0$ - 94,0) vs 43,8U $(33,0$ - 64,0), $p<0,0125$. Apesar da DDTI se manter sempre em doses inferiores comparativamente com as necessidades diárias de insulina pré-PSCI, não se verificaram diferenças estatisticamente significativas durante o restante seguimento.

No que diz respeito às complicações, no período de 20 anos, verificou-se a existência de dois episódios de hipoglicemia severa $(0,0095$ episódios/doente/ano) e cinco episódios de cetoacidose diabética (0,0238 episódios/doente/ ano). Não foi detetada nenhuma situação de infeção recorrente do local de inserção do cateter, com necessidade de antibiótico.

\section{DISCUSSÃO}

Tal como demonstram os resultados, o presente estudo obteve uma redução significativa da $\mathrm{HbA} 1 \mathrm{c}$ nos primeiros 10 anos de terapêutica com PSCl. A maior redução da HbA1c ocorreu nos primeiros seis meses de terapêutica $(2,1 \% ; p<0,0125)$, sendo superior à calculada noutros estudos semelhantes a este (cerca de 1,35\%).4,13 Dos 20 
18,0

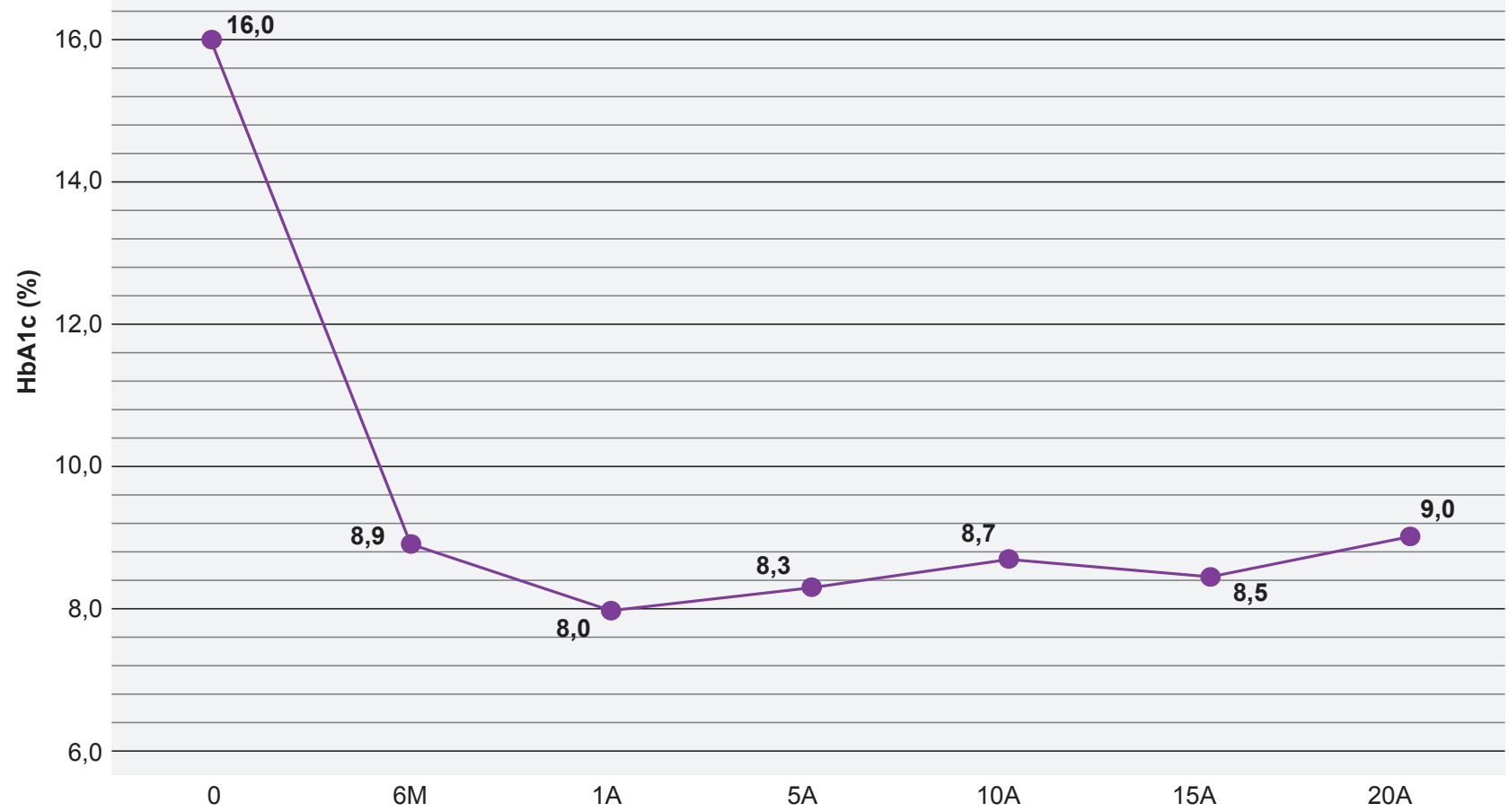

Figura 2 - Evolução da HbA1c do caso com 20 anos de $\mathrm{PSCl}(\mathrm{n}=1)$

PSCI: perfusão subcutânea contínua de insulina; M: meses; A: anos

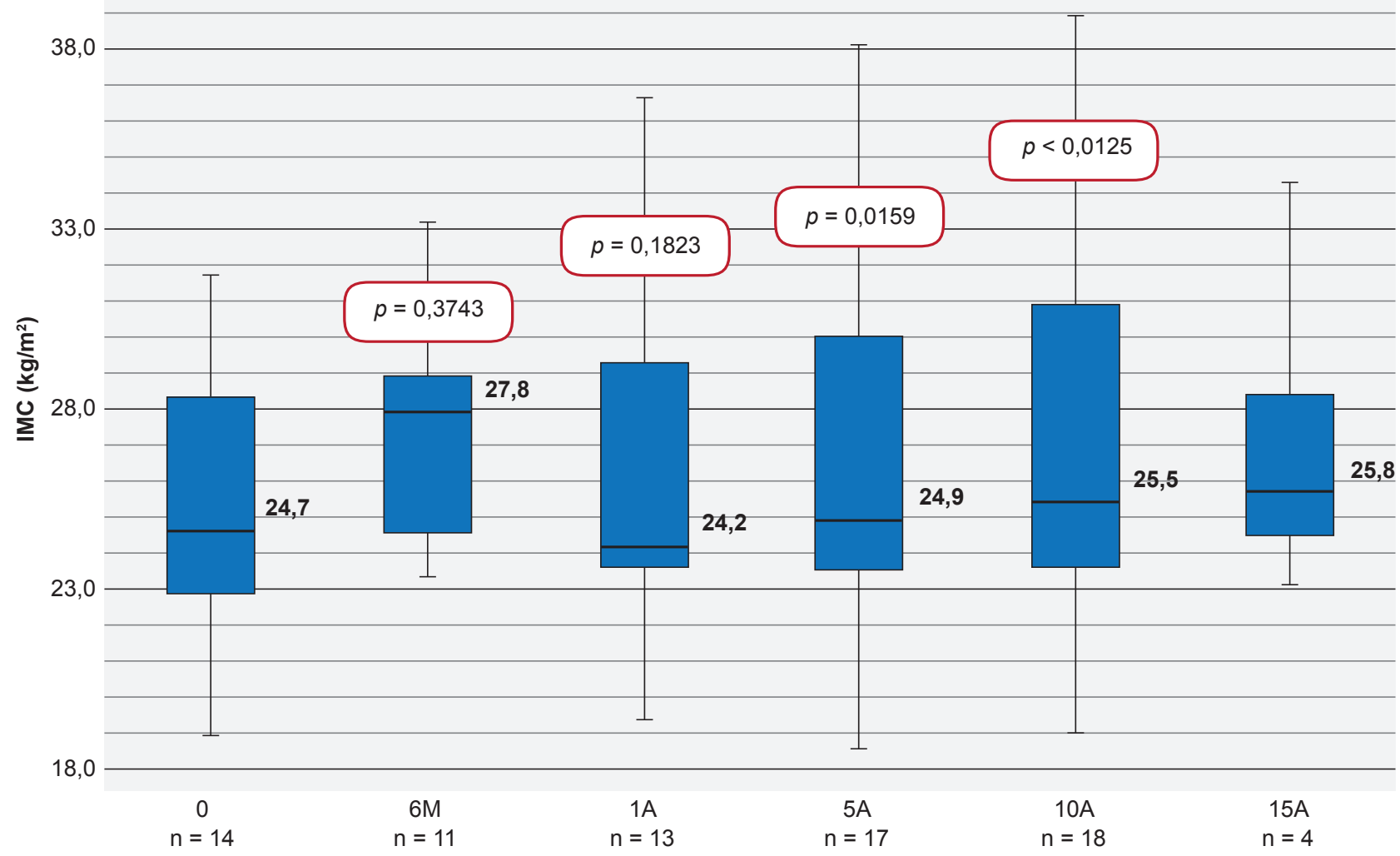

Figura 3 - Boxplots - Evolução do IMC. Estatisticamente significativo se $p<0,0125$. IMC: índice de massa corporal: M: meses; A: anos 


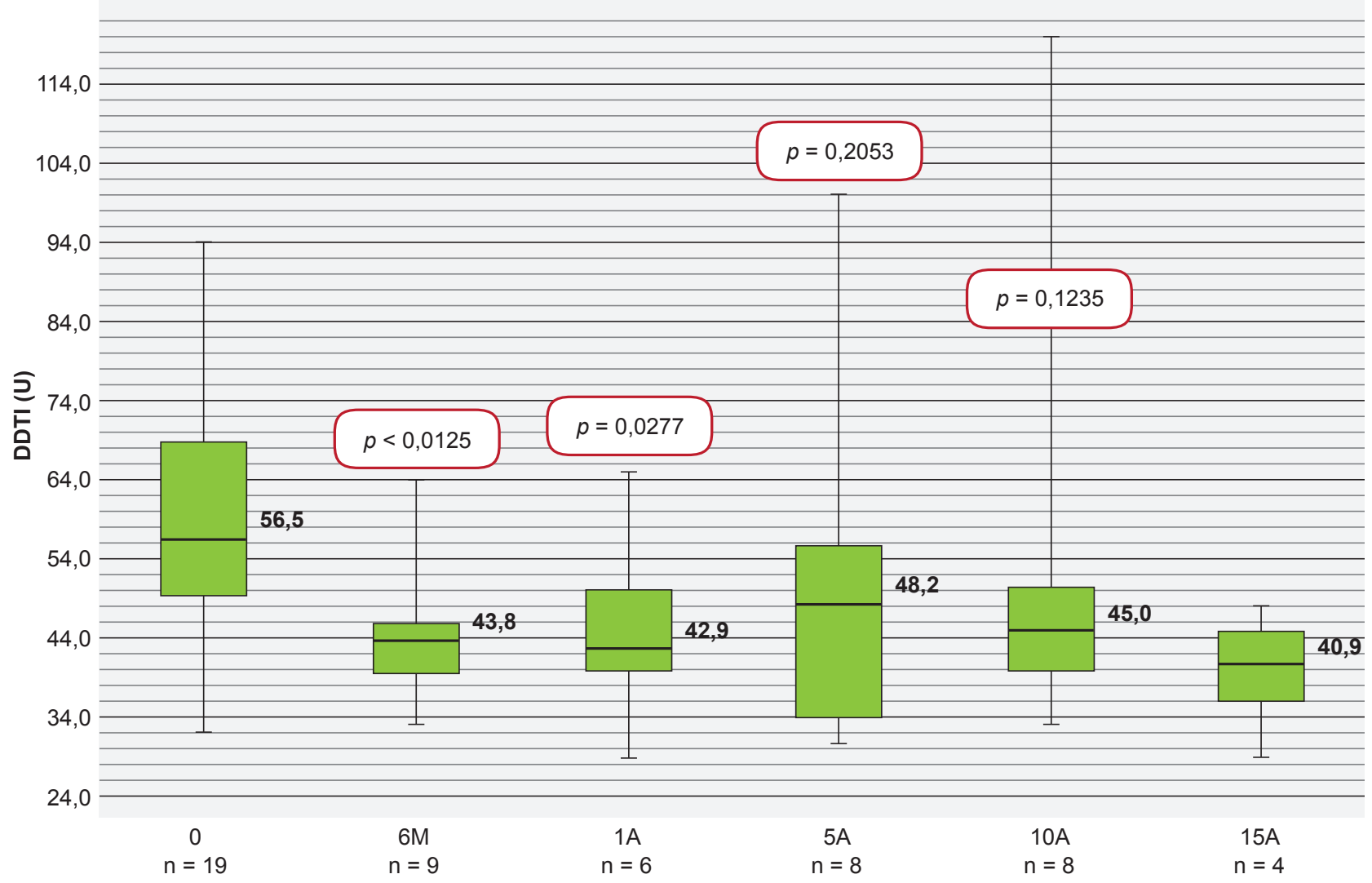

Figura 4 - Boxplots - Evolução da DDTI. Estatisticamente significativo se $p<0,0125$.

DDTI: dose diária total de insulina; M: meses; A: anos

doentes, 15 (75\%) iniciaram terapêutica com PSCl devido a controlo metabólico não aceitável. Está demonstrado que doentes com pior controlo metabólico prévio beneficiam de uma maior redução da HbA1c após introdução de PSCl. ${ }^{18-}$ ${ }^{20}$ Após seis meses de terapêutica com PSCl verificou-se uma ligeira subida da $\mathrm{HbA1c}$ atingindo um máximo de $7,6 \%$ ao fim do primeiro e quinto anos de seguimento. Outros estudos reportaram uma tendência para aumento da HbA1c após os primeiros seis meses de terapêutica com $\mathrm{PSCl}^{4}, 13,15,16 \mathrm{~A}$ perda de empenho e motivação à medida que o fator 'novidade' do sistema de perfusão se desvanece com o tempo, poderá justificar um agravamento do controlo glicémico a longo-prazo. A evolução da doença e o método de acompanhamento destes doentes, com um menor número de consultas/ano, também poderão ter tido impacto no controlo da glicemia relacionando-se com um pior controlo metabólico.

Após 10 anos de seguimento há uma redução significativa do tamanho da amostra $(n=4$ aos 15 anos e $n=$ 1 aos 20 anos), impossibilitando uma análise estatística consistente neste período. $O$ doente com maior tempo de acompanhamento na nossa instituição teve uma redução da $\mathrm{HbA} 1 \mathrm{c}$ de $16 \%$ para $9 \%$ ao fim de 20 anos de terapêutica com PSCl (Fig. 2). Qualquer redução da HbA1c observada nestes doentes é de extrema importância visto que uma pequena redução implica uma diminuição do risco de desenvolvimento de complicações, principalmente micro- vasculares, associadas à diabetes. ${ }^{2,3}$

Uma das preocupações da insulinoterapia intensiva é 0 aumento do peso. ${ }^{21} \mathrm{O}$ presente estudo avaliou a variação do IMC que se relaciona diretamente com a variação do peso. Verificou-se um aumento do IMC, especialmente nos primeiros seis meses, atingindo, no entanto, significado estatístico apenas aos 10 anos após PSCl; $24,7 \mathrm{~kg} / \mathrm{m}^{2}$ $(18,9-31,8)$ vs $25,5 \mathrm{~kg} / \mathrm{m}^{2}(18,9-38,9), p<0,0125$. Estes resultados são diferentes dos reportados noutros estudos que evidenciam uma ligeira diminuição do peso, apesar de não terem obtido diferenças estatisticamente significativas. ${ }^{4,13}$ Nos restantes períodos a curva do IMC é muito variável com períodos de subida e descida, sem qualquer variação significativa. O aumento do IMC ao longo do seguimento encontrado no nosso estudo poderá estar relacionado com o deficiente controlo glicémico que os doentes apresentavam quando iniciaram PSCl. Uma vez que na nossa amostra quinze $(75 \%)$ dos doentes iniciaram PSCl por hiperglicemia persistente, é possível que a melhoria do controlo glicémico verificado possa ter provocado um maior aumento de peso do que o verificado noutras séries em que o valor médio de $\mathrm{HbA} 1 \mathrm{c}$ à entrada estava mais próximo do objetivo, traduzindo-se num aumento do IMC.4,14,16

Relativamente à DDTI, verificou-se uma redução significativa nos primeiros seis meses de seguimento; $56,5 \mathrm{U}$ $(32,0$ - 94,0) vs $43,8 \cup(33,0-64,0), p<0,0125$. Nos restantes períodos o mesmo não aconteceu, no entanto importa 
referir que em nenhum momento a DDTI supera o valor prévio à $\mathrm{PSCl}$. Estes dados são consistentes com outros

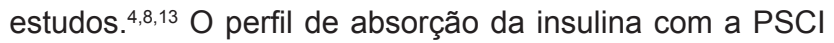
pode justificar a diminuição das necessidades diárias de insulina visto que este sistema permite uma absorção mais fisiológica e um ajuste mais preciso do débito de insulina basal nas 24 horas. . $^{43}$

Sobre as complicações agudas associadas à terapêutica com PSCl, este estudo confirma a existência de frequência reduzida de cetoacidose e hipoglicemia severa com PSCl, observada também em outros estudos. ${ }^{13,17}$ Karges et a/22 referem que o uso de análogos de insulina de ação rápida com a $\mathrm{PSCl}$ permite uma maior flexibilidade terapêutica com menor variabilidade glicémica, levando a menores taxas de complicações agudas e a longo-prazo, incluindo hipoglicemia. A redução do risco de cetoacidose diabética com PSCl foi associado a uma auto- monitorização da glicemia mais frequente. ${ }^{22} \mathrm{~A}$ maioria dos estudos mostra que, com educação e prática no uso do sistema de perfusão, a frequência de cetoacidose é similar tanto na $\mathrm{PSCl}$ como na MAD. ${ }^{13}$ Importa referir que a taxa de complicações, nomeadamente de hipoglicémia severa, observadas no presente estudo, podem estar subvalorizadas devido à possibilidade de ausência de registos relativos a esta informação.

As limitações deste estudo prendem-se essencialmente com o facto de se tratar de um estudo retrospetivo. A obtenção de dados relativamente ao IMC e DDTI foi limitada, o que levou a uma redução da capacidade de inferência estatística nestas variáveis. Por se tratar de uma tecnologia relativamente recente, são poucos os doentes com DM1 a serem tratados com PSCI há mais de 10 anos. Isto limita a análise estatística, não impedindo, no entanto, uma análise descritiva do comportamento de doentes reais a beneficia- rem desta terapêutica a longo prazo.

Apesar das limitações, este estudo reflete a prática clínica diária de uma população heterogénea com DM1 a usufruir de terapêutica com PSCl.

\section{CONCLUSÃO}

A evidência sugere que a terapêutica com $\mathrm{PSCl}$ é efetivamente vantajosa no controlo metabólico em doentes com DM1 selecionados. No nosso estudo foi possível confirmar que o efeito benéfico da $\mathrm{PSCl}$ pode ser sustentado por longos períodos de tempo, com um baixo risco de complicações agudas associadas. Esta forma de tratamento poderá, no futuro, ser considerada como tratamento de primeira linha em pessoas com DM1.

\section{PROTECÇÃO DE PESSOAS E ANIMAIS}

Os autores declaram que os procedimentos seguidos estavam de acordo com os regulamentos estabelecidos pelos responsáveis da Comissão de Investigação Clínica e Ética e de acordo com a Declaração de Helsínquia da Associação Médica Mundial.

\section{CONFIDENCIALIDADE DOS DADOS}

Os autores declaram ter seguido os protocolos do seu centro de trabalho acerca da publicação de dados.

\section{CONFLITOS DE INTERESSE}

Os autores declaram não terem qualquer conflito de interesse relativamente ao presente artigo.

\section{FONTES DE FINANCIAMENTO}

Os autores declaram não ter recebido subsídios ou bolsas para a elaboração do artigo.

\section{REFERÊNCIAS}

1. Expert Committee on the Diagnosis and Classification of Diabetes Mellitus. Report of the expert committee on the diagnosis and classification of diabetes mellitus. Diabetes Care. 2003;26:S5-20.

2. The Diabetes Control and Complications Trial Research Group. The effect of intensive treatment of diabetes on the development and progression of long-term complications in insulin-dependent diabetes mellitus. N Engl J Med. 1993;329:977-86.

3. Epidemiology of Diabetes Interventions and Complications (EDIC). Design, implementation, and preliminary results of a long-term follow-up of the Diabetes Control and Complications Trial cohort. Diabetes Care. 1999;22:99-111.

4. Balsa AM, Neves C, Alves M, Pereira M, Carvalho D, Medina JL. Terapêutica de infusão subcutânea contínua de insulina. Acta Med Port. 2011;24:147-56.

5. Didangelos T, Iliadis F. Insulin pump therapy in adults. Diab Res Clin Practice. 2011;93:S109-13

6. Pickup $\mathrm{J}$, Keen $\mathrm{H}$. Continuous subcutaneous insulin infusion at 25 years: evidence base for the expanding use of insulin pump therapy in type 1 diabetes. Diabetes Care. 2002;25:593-8.

7. Direção Geral de Saúde. Circular normativa n¹7/DSCS/DGID. Gestão integrada de diabetes - elegibilidade dos doentes para tratamento através de perfusão subcutânea contínua de insulina. Lisboa: DGS; 2008.

8. Pozzilli P, Battelino T, Danne T, Hovorka R, Jarosz-Chobot P, Renard E. Continuous subcutaneous insulin infusion in diabetes: patient populations, safety, efficacy, and pharmacoeconomics. Diabetes Metab Res Rev. 2016;32:21-39.

9. Carlsson BM, Attvall S, Clements M, Gumpeny SR, Pivodic A Sternemalm L, et al. Insulin pump-long-term effects on glycemic control: an observational study at 10 diabetes clinics in Sweden. Diabetes Technol Ther. 2013;15:302-7.

10. Orr CJ, Hopman W, Yen JL, Houlden RL. Long-term efficacy of insulin pump therapy on glycemic control in adults with type 1 diabetes mellitus. Diabetes Technol Ther. 2015;17:49-54.

11. Marchand L, Kawasaki-Ogita Y, Place J, Fayolle C, Lauton D, Boulet F, et al. Long-term effects of continuous subcutaneous insulin infusion on glucose control and microvascular complications in patients with type 1 diabetes. J Diabetes Sci Technol. 2017;11:924-9.

12. Nixon R, Folwell R, Pickup JC. Variations in the quality and sustainability of long-term glycaemic control with continuous subcutaneous insulin infusion. Diabet Med. 2014;21:1174-7.

13. Saraiva J, Carrilho F, Barros L, Baptista C, Melo M, Gomes L, et al. Long term follow-up in type 1 diabetes patients using continuous subcutaneous insulin infusion systems. Infusystems International. 2013;12:6-8.

14. Esteves C, Belo S, Neves MC, Arteiro C, Carvalho D. Glycemic control and weight outcomes after initiation of continuous subcutaneous insulin infusion therapy. Rev Port Endocrinol Diabetes Metabol. 2015;10:12832.

15. Santos MJ. Avaliação da qualidade de vida e do controlo glicémico em diabéticos tipo $1 \mathrm{com}$ bomba infusora de insulina. Tese de Mestrado em Medicina. Porto: Instituto de Ciências Biomédicas Abel Salazar, Universidade do Porto; 2009.

16. Monteiro AM, Alves M, Pereira ML, Marques O. Experiência do serviço 
de endocrinologia do hospital de Braga no tratamento de diabéticos tipo 1 com bombas infusoras de insulina. In: Hospital de Bragaendocrinologia, diabetes e metabolismo - comunicações e conferências. Braga: Hospital de Braga; 2015.

17. Joubert M, Morera J, Vicente A, Rod A, Parienti JJ, Reznik Y. Crosssectional survey and retrospective analysis of a large cohort of adults with type 1 diabetes with long-term continuous subcutaneous insulin infusion treatment. J Diabetes Sci Technol. 2014;8:1005-10.

18. Pickup JC, Sutton AJ. Severe hypoglycaemia and glycaemic control in Type 1 diabetes: meta-analysis of multiple daily insulin injections compared with continuous subcutaneous insulin infusion. Diabet Med. 2008;25:765-74

19. Pickup JC, Kidd J, Burmiston S, Yemane N. Determinants of glycaemic control in type 1 diabetes during intensified therapy with multiple daily insulin injections or continuous subcutaneous insulin infusion: importance of blood glucose variability. Diabetes Metab Res Rev. 2006;22:232-7.

20. Retnakaran R, Hochman J, DeVries JH, Hanaire-Broutin H, Heine RJ, Melki V, et al. Continuous subcutaneous insulin infusion versus multiple daily injections: the impact of baseline A1c. Diabetes Care. 2004;27:2590-6.

21. Weight gain associated with intensive therapy in the diabetes contro and complications trial. Diabetes Care. 1988;11:567-73.

22. Karges B, Schwandt A, Heidtmann B, Kordonouri O, Binder E, Schierloh $U$, et al. Association of insulin pump therapy vs insulin injection therapy with severe hypoglycemia, ketoacidosis, and glycemic control among children, adolescents, and young adults with type 1 diabetes. JAMA. 2017;318:1358-66. 